\title{
Comparison of three methods for 25-hydroxyvitamin D analysis in umbilical cord serum
}

\author{
J. Y. Zhang ${ }^{1}$, M. Kinsella ${ }^{1}$, K. Sarafin ${ }^{2}$, S. P. J. Brooks ${ }^{2}$, A. J. Lucey ${ }^{1}$, K. D. Cashman ${ }^{1}$ and M. Kiely $^{1}$ \\ ${ }^{1}$ Vitamin D Research Group, School of Food and Nutritional Sciences, University College Cork, Ireland and ${ }^{2}$ Nutrition \\ Research Division, Health Canada, Ottawa, Canada
}

Variability among estimates of serum 25-hydroxyvitamin D (25OHD) concentrations between different analytical methods and laboratories is well documented ${ }^{(1)}$. There is evidence to suggest that commercial assays produce more unreliable estimates at the higher and lower ends of the distribution of $25 \mathrm{OHD}$ concentrations, due to higher limits of detection and cross-reactivity ${ }^{(2)}$. Liquid chromatographytandem mass spectrometry (LC-MS/MS) is regarded as a gold standard reference method when NIST standard reference materials (SRM) are applied ${ }^{(3)}$. As umbilical cord serum 25OHD concentrations are typically lower than adult values, the aim of this study was to compare three commonly used analytical methods to measure total $25 \mathrm{OHD}$ concentrations in 86 cord sera that were stored at $-80^{\circ} \mathrm{C}$. Total serum 25OHD concentrations were quantified using an enzyme immunoassay (EIA), Immuno Diagnostic Systems (IDS), Boldon, UK, a direct competitive chemiluminescence immunoassay (LIAISON TOTAL Assay), Diasorin Stillwater, USA, and an LC-MS/MS method, which is traceable to the US National Institute for Standards and Technology (NIST) higher order reference measurement procedure ${ }^{(4)}$. Data were compared using the Bland-Altman method.

Both IDS EIA and DiaSorin Liaison correlated strongly with LC-MS/MS ( $r=0.945$ and 0.976 , both $P<0.001$ ). However, substantial bias was evident in the data from both methods, which ranged from $-20 \%$ to $176 \%$ using IDS EIA and $-20 \%$ to $193 \%$ with DiaSorin Liaison, respectively and the bias was highest at low 25OHD concentrations. The mean difference between IDS EIA and LC-MS/MS was $6.8 \mathrm{nmol} / \mathrm{L}$ and between DiaSorin Liaison and LC-MS/MS was $9.9 \mathrm{nmol} / \mathrm{L}$ (both $P<0.001$ ), see Table. IDS EIA and DiaSorin Liaison over-estimated total $25 \mathrm{OHD}$ by 35 and $41 \%$, respectively, see Figure.

\begin{tabular}{|c|c|c|c|c|c|c|c|}
\hline & Mean & SD & Median & 25 th & 75th & Min & Max \\
\hline LC-MS/MS & 27.0 & 14.8 & 24.5 & 17.3 & 32.5 & 5.2 & 84 \\
\hline IDS EIA & 33.8 & 14.8 & 30.6 & 23.2 & 39.4 & 13.8 & \\
\hline DiaSorin Liaison Total & 36.9 & 19.1 & 32.1 & 22.7 & 43.4 & 10.0 & \\
\hline
\end{tabular}
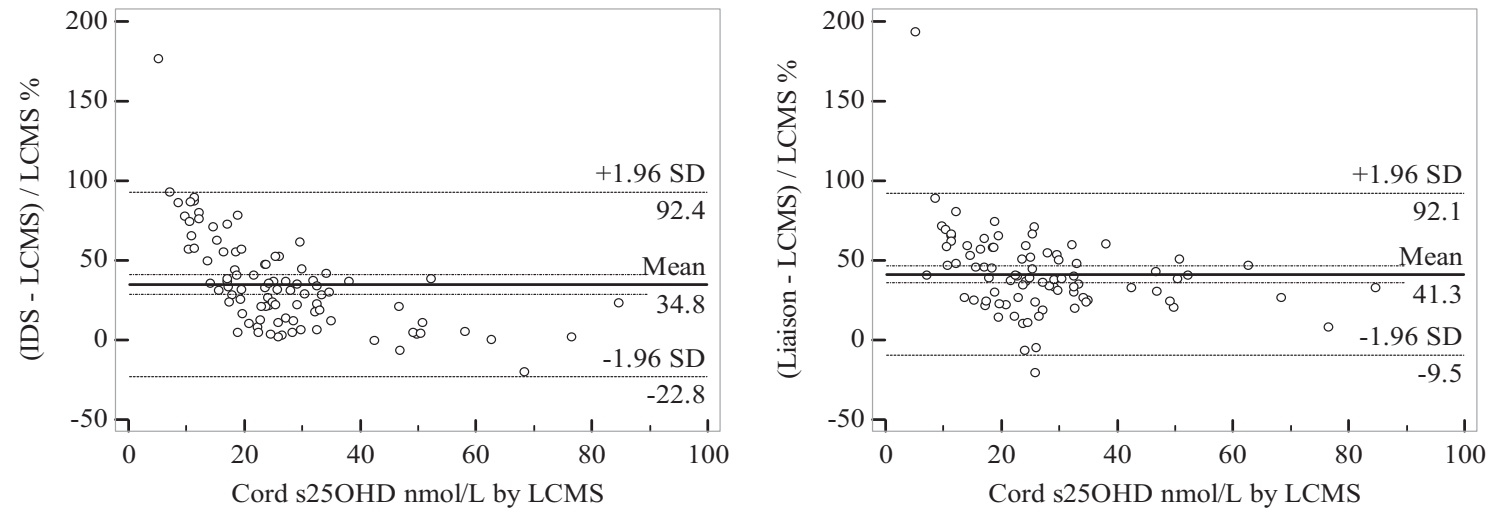

Large differences were observed in the concentrations of serum 25OHD in cord samples using both the IDS EIA and DiaSorin Liaison methods compared with LC-MS/MS. Reported levels of serum 25OHD concentrations in umbilical cord samples are not comparable between methods due to the positive bias observed and their validity is questionable, particularly at the low concentrations of $25 \mathrm{OHD}$ typically observed in cords. This study re-emphasises the need for international standardisation of serum 25OHD measurements, such as the ongoing Vitamin D Standardisation Program (VDSP) ${ }^{(5)}$.

Supported by the Higher Education Authority Program for Research in Third Level Institutions (PRTLI Cycle 4)

1. Roth HJ, Schmidt-Gayk H, Weber H et al. (2008) Ann Clin Biochem 45, 153-159.

2. Lind C, Chen J, Byrjalsen I (1997). Clinical chemistry 43, 943-949.

3. Yates AM, Bowron A, Calton L et al. (2008) Clin Chem 54, 2082-2084.

4. Tai SS, Bedner M, Phinney KW (2010) Anal Chem 82, 1942-1948.

5. Sempos CT, Vesper HW, Phinney KW et al. (2012) Scand J Clin Lab Invest Suppl 243, 32-40. 